\title{
Variational Iteration Method for Solving Differential Equations with Piecewise Constant Arguments
}

\author{
Qi Wang a,", Fenglian Fu ${ }^{\text {b }}$ \\ ${ }^{a}$ Faculty of Applied Mathematics, Guangdong University of Technology, Guangzhou, Guangdong 510006, \\ China \\ ${ }^{b}$ Faculty of Environmental Science and Engineering, Guangdong University of Technology, Guangzhou, \\ Guangdong 510006, China
}

\begin{abstract}
In this paper, variational iteration method is applied for finding the solution of differential equations with piecewise constant arguments. A correction functional is constructed by a general Lagrange multiplier, which can be identified by variational theory. This technique provides a sequence of functions which converges to the exact solution of the problem without discretization of the variables. The flexibility and adaptation provided by the method have been verified by an example.
\end{abstract}

Index Terms: Variational iteration method; Piecewise constant arguments; Approximate analytical solution

(C) 2012 Published by MECS Publisher. Selection and/or peer review under responsibility of the Research Association of Modern Education and Computer Science.

\section{Introduction}

Recently, there has been much research concerning properties of solutions of differential equations with piecewise constant arguments (EPCA) [1-4]. In these equations, the derivatives of the unknown functions depend on not just the time $\boldsymbol{t}$ before $\boldsymbol{t}$. Since this is essentially expressing the derivatives on terms of the solution at discrete points of time before $\boldsymbol{t}$, it is usually referred to as a hybrid system, other examples of the application of these equations to the problems of biology, cellular neural networks and mechanical systems can be found in [5-8].

EPCA has been under intensive investigation for the last twenty years. The first studies in this field have been given in $[9,10]$, after this, stability, contractivity and existence of periodic solutions have been treated by several authors, see [11-13] and references therein. The general theory and basic results for EPCA have been thoroughly investigated in the book of Wiener[14]. Nowadays, much research has been focused on the

* Corresponding author.

E-mail address: bmwzwq@126.com 
numerical solutions of EPCA, including convergence[15], stability[16] and oscillation[17]. However, we can't find any results concerning the approximate anaytical solution of EPCA have been published. In our paper, we will apply the well-known anaytical approximate technique: the variational iteration method to the follwing EPCA:

$$
\left\{\begin{array}{l}
u^{\prime}(t)=a u(t)+b u\left(\left[\frac{t}{2}\right]\right), \quad t \geq 0, \\
u(0)=u_{0},
\end{array}\right.
$$

where $a, b, u_{0} \in R$ and [.] denotes the greatest integer function. The main goal of this paper is to extend the variational iteration method to find the solution of (1).

The variational iteration method (VIM) [18-21], which is a well-established technique with wide applications for ordinary differential equations, partial differential equations and delay differential equations, etc. More materials of the classical solution techniques that are most commonly used to solve equations and systems, such as the Adomian decomposition method (ADM) are found in [22], Differential transformation method (DTM) [23], Homotopy analysis method (HAM) [24] and the Homotopy perturbation method (HPM) [25]. The VIM is the most effective and convenient one for both weakly and strongly nonlinear equations. This method has been shown to effectively, easily, and accurately solve a large class of linear and nonlinear problems.

\section{PrelimInaries}

Definition 1[14] A solution of (1) on $[0, \infty)$ is a function $u(t)$ that satisfies the conditions:

(i) $u(t)$ is continuous on $[0, \infty)$,

(ii) The derivative $\boldsymbol{u}^{\prime}(\boldsymbol{t})$ exists at each point $t \in[0, \infty)$, with the possible exception of the point $t=2 n$, $n=0,1, \cdots$, where one-sided derivatives exist,

(iii) Eq. (1) is satisfied on each interval $[2 n, 2(n+1)) \subset[0, \infty)$.

Theorem 1 [14]. If $a<0,|b|<-a$, then the solution of (1) tends to zero as $t \rightarrow \infty$ for any given $u_{0}$

\section{VARIATIONAL ITERATION MRTHOD (VIM)}

The VIM is the general Lagrange method, in which an extremely accurate approximation at some special point can be obtained, but not an analytical solution. To illustrate the basic idea of the VIM we consider the following general differential equation

$$
L u+N u=g(x)
$$

where $L$ and $N$ are linear and nonlinear operator, respectively, and $g(x)$ is the inhomogeneous term. In [26-29], the author proposed the VIM where a correction functional for (2) can be written as

$$
u_{n+1}(x)=u_{n}(x)+\int_{0}^{x} \lambda(t)\left[L u_{n}(t)+N u_{n}(t)-g(t)\right] d t
$$

where $\lambda$ is a general Lagrange's multiplier, which can be identified optimally via the variational theory, and $u_{n}$ as a restricted variation which means $\delta u_{n}=0$. It is to be noted that the Lagrange multiplier $\lambda$ can be a constant or a function. 
The VIM should be employed by following two main steps. It is required first to determine the Lagrange multiplier $\lambda$ that can be identified optimally via integration by parts and by using a restricted variation. Having $\lambda$ determined, an iteration formula, without restricted variation, should be used for the determination of the successive approximations $u_{n+1}(x)$ of the solution $u(x)$. The zeroth approximation $u_{0}$ can be any selective function. Consequently, the solution is given by

$$
u(x)=\lim _{n \rightarrow \infty} u_{n}(x) \text {. }
$$

Now, we apply the VIM to (1). A correction functional is constructed as follows:

$$
u_{n+1}(t)=u_{n}(t)+\int_{0}^{t} \lambda(s)\left[u_{n}^{\prime}(s)-a u_{n}(s)-b u_{n}\left(\left[\frac{s}{2}\right]\right)\right] d s .
$$

Taking variational on both sides of (5), we have

$$
\begin{aligned}
\delta u_{n+1}(t) & =\delta u_{n}(t)+\delta \int_{0}^{t} \lambda(s) u_{n}^{\prime}(s) d s, \\
& =\delta u_{n}(t)+\lambda(t) \delta u_{n}(t)-\int_{0}^{t} \lambda^{\prime}(s) \delta u_{n}(s) d s, \\
& =(1+\lambda(t)) \delta u_{n}(t)-\int_{0}^{t} \lambda^{\prime}(s) \delta u_{n}(s) d s,
\end{aligned}
$$

this yields the stationary conditions:

$$
\left\{\begin{array}{l}
1+\lambda(t)=0, \\
\left.\lambda^{\prime}(s)\right|_{s=t}=0 .
\end{array}\right.
$$

Thus

$$
\lambda(s)=-1
$$

and we obtain the following iteration formula

$$
u_{n+1}(t)=u_{n}(t)-\int_{0}^{t}\left[u_{n}^{\prime}(s)-a u_{n}(s)-b u_{n}\left(\left[\frac{s}{2}\right]\right)\right] d s .
$$

So we have

$$
\begin{aligned}
& u_{0}(t)=u_{0}, \\
& u_{1}(t)=u_{0}(t)-\int_{0}^{t}\left[u_{0}^{\prime}(s)-a u_{0}(s)-b u_{0}\left(\left[\frac{s}{2}\right]\right)\right] d s, \\
& u_{2}(t)=u_{1}(t)-\int_{0}^{t}\left[u_{1}^{\prime}(s)-a u_{1}(s)-b u_{1}\left(\left[\frac{s}{2}\right]\right)\right] d s, \\
& u_{3}(t)=u_{2}(t)-\int_{0}^{t}\left[u_{2}^{\prime}(s)-a u_{2}(s)-b u_{2}\left(\left[\frac{s}{2}\right]\right)\right] d s, \\
& u(t)=\lim _{n \rightarrow \infty} u_{n}(t) .
\end{aligned}
$$

In order to overcome the difficulty from the greatest integer function $[\cdot]$, we can consider the above iteration formula in a series of intervals: $[0,2),[2,4),[4,6), \ldots,[2 n, 2(n+1))$ where $n=0,1,2, \ldots$ Following this way, each integral in iteration formulas will be easily computed.

The above analysis yields the following theorem:

Theorem 2. The VIM solution of (1) can be determined by (9) with the iterations (8). 
In the next section, the VIM is successfully applied for solving a linear EPCA.

\section{APPLICATION}

In what follows, we will apply the VIM method to a physical model to illustrate the strength of the method.

Let $a=2, b=-3$ and $u_{0}=1$ in (1), this in turn gives the successive approximations by (8).

When $t \in[0,2)$,

$$
\begin{aligned}
u_{1,0}(t) & =1 \\
u_{1,1}(t) & =u_{1,0}(t)-\int_{0}^{t}\left[u_{1,0}^{\prime}(s)-2 u_{1,0}(s)+3 u_{1,0}\left(\left[\frac{s}{2}\right]\right)\right] d s \\
& =1-\int_{0}^{t}(-2+3) d s \\
& =1-t \\
u_{1,2}(t) & =u_{1,1}(t)-\int_{0}^{t}\left[u_{1,1}^{\prime}(s)-2 u_{1,1}(s)+3 u_{1,1}\left(\left[\frac{s}{2}\right]\right)\right] d s \\
& =1-t-\int_{0}^{t}\left[-1-2(1-s)+3 u_{1,1}(0)\right] d s \\
& =1-t-t^{2}, \\
u_{1,3}(t) & =u_{1,2}(t)-\int_{0}^{t}\left[u_{1,2}^{\prime}(s)-2 u_{1,2}(s)+3 u_{1,2}\left(\left[\frac{s}{2}\right]\right)\right] d s \\
& =u_{1,2}(t)-\int_{0}^{t}\left[-1-2 s-2\left(1-s-s^{2}\right)+3 u_{1,2}(0)\right] d s=1-t-t^{2}-\frac{2}{3} t^{3}, \\
u_{1,4}(t) & =u_{1,3}(t)-\int_{0}^{t}\left[u_{1,3}^{\prime}(s)-2 u_{1,3}(s)+3 u_{1,3}\left(\left[\frac{s}{2}\right]\right)\right] d s \\
& =u_{1,3}(t)-\int_{0}^{t}\left[-3+\frac{4}{3} s^{3}+3 u_{1,3}(0)\right] d s \\
& =1-t-t^{2}-\frac{2}{3} t^{3}-\frac{1}{3} t^{4},
\end{aligned}
$$

When $t \in[2,4)$,

$$
\begin{aligned}
& u_{2,0}(t)=1 \text {, } \\
& u_{2,1}(t)=u_{2,0}(t)-\int_{0}^{t}\left[u_{2,0}^{\prime}(s)-2 u_{2,0}(s)+3 u_{2,0}\left(\left[\frac{s}{2}\right]\right)\right] d s \\
& =1-\int_{0}^{t}(-2+3) d s \\
& =1-t \text {, } \\
& u_{2,2}(t)=u_{2,1}(t)-\int_{0}^{t}\left[u_{2,1}^{\prime}(s)-2 u_{2,1}(s)+3 u_{2,1}\left(\left[\frac{s}{2}\right]\right)\right] d s
\end{aligned}
$$




$$
\begin{aligned}
& =1-t-\int_{0}^{t}\left[-1-2(1-s)+3 u_{2,1}(1)\right] d s \\
& =1+2 t-t^{2} \\
u_{2,3}(t) & =u_{2,2}(t)-\int_{0}^{t}\left[u_{2,2}^{\prime}(s)-2 u_{2,2}(s)+3 u_{2,2}\left(\left[\frac{s}{2}\right]\right)\right] d s \\
& =u_{2,2}(t)-\int_{0}^{t}\left[2-2 s-2\left(1+2 s-s^{2}\right)+3 u_{2,2}(1)\right] d s \\
& =1-4 t+2 t^{2}-\frac{2}{3} t^{3}, \\
u_{2,4}(t) & =u_{2,3}(t)-\int_{0}^{t}\left[u_{2,3}^{\prime}(s)-2 u_{2,3}(s)+3 u_{2,3}\left(\left[\frac{s}{2}\right]\right)\right] d s \\
& =u_{2,3}(t)-\int_{0}^{t}\left[-6+12 s-6 s^{2}+\frac{4}{3} s^{3}+3 u_{2,3}(1)\right] d s \\
& =1+7 t-4 t^{2}+\frac{4}{3} t^{3}-\frac{1}{3} t^{4},
\end{aligned}
$$

When $t \in[4,6)$,

$$
\begin{aligned}
u_{3,0}(t) & =1, \\
u_{3,1}(t) & =u_{3,0}(t)-\int_{0}^{t}\left[u_{3,0}^{\prime}(s)-2 u_{3,0}(s)+3 u_{3,0}\left(\left[\frac{s}{2}\right]\right)\right] d s \\
& =1-\int_{0}^{t}(-2+3) d s \\
& =1-t \\
u_{3,2}(t) & =u_{3,1}(t)-\int_{0}^{t}\left[u_{3,1}^{\prime}(s)-2 u_{3,1}(s)+3 u_{3,1}\left(\left[\frac{s}{2}\right]\right)\right] d s \\
& =u_{3,1}(t)-\int_{0}^{t}\left[-1-2(1-s)+3 u_{3,1}(2)\right] d s \\
& =1+5 t-t^{2}, \\
u_{3,3}(t) & =u_{3,2}(t)-\int_{0}^{t}\left[u_{3,2}^{\prime}(s)-2 u_{3,2}(s)+3 u_{3,2}\left(\left[\frac{s}{2}\right]\right)\right] d s \\
& =u_{3,2}(t)-\int_{0}^{t}\left[5-2 s-2\left(1+5 s-s^{2}\right)+3 u_{3,2}(2)\right] d s=1-19 t+5 t^{2}-\frac{2}{3} t^{3}, \\
u_{3,4}(t) & =u_{3,3}(t)-\int_{0}^{t}\left[u_{3,3}^{\prime}(s)-2 u_{3,3}(s)+3 u_{3,3}\left(\left[\frac{s}{2}\right]\right)\right] d s \\
& =u_{3,3}(t)-\int_{0}^{t}\left[-21+48 s-12 s^{2}+\frac{4}{3} s^{3}+3 u_{3,3}(2)\right] d s \quad=1+69 t-19 t^{2}+\frac{10}{3} t^{3}-\frac{1}{3} t^{4},
\end{aligned}
$$

In a word, in the interval $[2 n, 2(n+1)), n=0,1,2$, we have the following iteration formulas: 


$$
\begin{aligned}
u_{n+1,0}(t) & =1 \\
u_{n+1, m}(t) & =u_{n+1, m-1}(t) \\
& -\int_{0}^{t}\left[u_{n+1, m-1}^{\prime}(s)-2 u_{n+1, m-1}(s)+3 u_{n+1, m-1}(n)\right] d s
\end{aligned}
$$

In view of (9), we can obtain the approximate analytical solution. Usually, the $m+1$ th approximation is used for numerical purposes.

In Table 1, we compare the absolute errors of the VIM $(m=4,5)$ with the ones of the 1-Gauss-Legendre method[16] using $h=0.02$. The graphs of the exact solution and 7th approximate solutions are shown in Fig. 1.

Table. 1.Comparison of the absolute errors

\begin{tabular}{|l|l|l|l|}
\hline \multirow{2}{*}{$\mathrm{t}$} & \multicolumn{2}{|c|}{ VIM } & \multirow{2}{*}{$\begin{array}{l}\text { 1-Gauss- Legendre } \\
\text { method }\end{array}$} \\
\cline { 2 - 4 } & \multicolumn{1}{|c|}{$\mathrm{m}=4$} & \multicolumn{1}{|c|}{$\mathrm{m}=5$} & \\
\hline 0.7 & $3.09 \mathrm{E}-6$ & $5.47 \mathrm{E}-7$ & $2.33 \mathrm{E}-4$ \\
\hline 1.4 & $4.14 \mathrm{E}-6$ & $7.72 \mathrm{E}-7$ & $4.16 \mathrm{E}-4$ \\
\hline 2.1 & $5.16 \mathrm{E}-6$ & $5.17 \mathrm{E}-6$ & $3.81 \mathrm{E}-3$ \\
\hline 2.8 & $4.34 \mathrm{E}-5$ & $6.14 \mathrm{E}-6$ & $5.42 \mathrm{E}-3$ \\
\hline 3.5 & $5.51 \mathrm{E}-5$ & $8.75 \mathrm{E}-6$ & $8.15 \mathrm{E}-3$ \\
\hline 4.2 & $7.36 \mathrm{E}-5$ & $1.08 \mathrm{E}-5$ & $1.96 \mathrm{E}-2$ \\
\hline 4.9 & $3.84 \mathrm{E}-4$ & $2.92 \mathrm{E}-5$ & $2.37 \mathrm{E}-2$ \\
\hline 5.6 & $8.26 \mathrm{E}-4$ & $6.52 \mathrm{E}-5$ & $4.73 \mathrm{E}-2$ \\
\hline
\end{tabular}



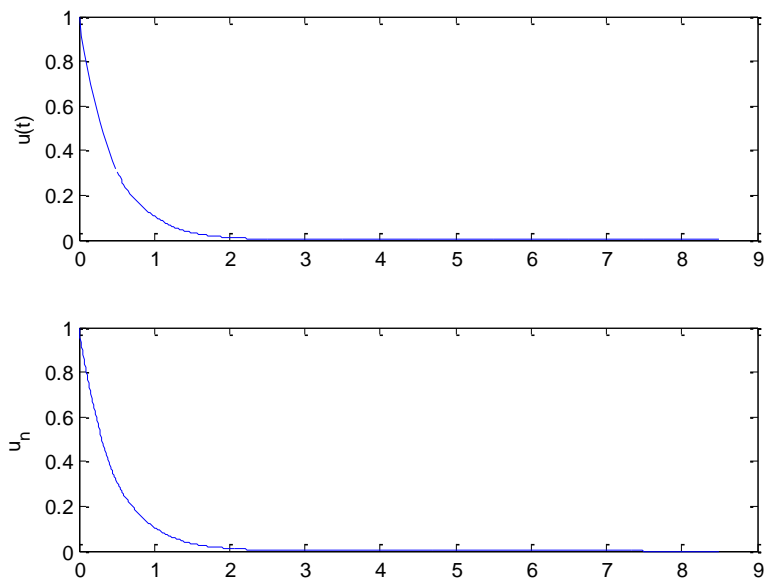

Fig. 1. Comparison of the exact solution with the VIM solution, Upper: exact solution, Lower: 7th VIM solution

\section{CONCLUSION}

In this paper, we have demonstrated the feasibility of the VIM for solving a EPCA model. We obtain highaccuracy approximate solutions. The numerical results also show that the VIM offers a very effective and convenient approach to the approximate solution of EPCA.

\section{References}

[1] G.Q. Wang, "Periodic solutions of a neutral differential equation with piecewise constant arguments," $J$. Math. Anal. Appl., vol. 326, pp. 736-747, 2007.

[2] M.U. Akhmet, "On the reduction principle for differential equations with piecewise constant argument of generalized type,” J. Math. Anal. Appl., vol. 336, pp. 646-663, 2007.

[3] M.U. Akhmet, "Asymptotic behavior of solutions of differential equations with piecewise constant arguments," Appl. Math. Lett.., vol. 21, pp. 951-956, 2008.

[4] M.U. Akhmet, "Stability of differential equations with piecewise constant arguments of generalized type," Nonlinear Anal., vol. 68, pp. 794-803, 2008.

[5] D. Altintan, "Extension of the logistic equation with piecewise constant arguments and population dynamics," M.Sc.Thesis, Middle East Technical University, 2006.

[6] K.L. Cooke and J. Weiner, "A survey of differential equation with piecewise continuous argument," Lecture Notes in Mathematics, Springer, Berlin, 1991.

[7] L. Dai and L. Fan, "Analytical and numerical approaches to characteristics of linear and nonlinear vibratory systems under piecewise discontinuous disturbances," Commun. Nonlinear Sci. Numer. Simul., vol. 9, pp. 417-429, 2004.

[8] F. Gurcan and F. Bozkurt, "Global stability in a population model with piecewise constant arguments," J. Math. Anal. Appl., vol. 360, pp. 334-342, 2009.

[9] K.L. Cooke and J. Wiener, "Retarded differential equations with piecewise constant delays," J. Math. Anal. Appl., vol. 99, pp. 265-297, 1984.

[10] S.M. Shah and J. Wiener, "Advanced differential equations with piecewise constant argument deviations,” Int. J. Math. Math. Sci., vol. 6, pp. 671-703, 1983. 
[11] A.F. Ivanov, "Global dynamics of a differential equation with piecewise constant argument," Nonlinear Anal., vol. 71, pp. e2384-e2389, 2009.

[12] Y. Muroya, "New contractivity condition in a population model with piecewise constant arguments," $J$. Math. Anal. Appl., vol. 346, pp. 65-81, 2008.

[13] Y.H. Xia, Z.K. Huang, and M.A. Han, "Existence of almost periodic solutions for forced perturbed systems with piecewise constant argument," J. Math. Anal. Appl., vol. 333, pp. 798-816, 2007.

[14] J. Wiener, Generalized Solutions of Functional Differential Equations, Singapore: World Scientific, 1993.

[15] M.H. Song, Z.W. Yang, and M.Z. Liu, "Stability of $\boldsymbol{\theta}$-methods for advanced differential equations with piecewise continuous arguments," Comput.Math. Appl., vol. 49, pp. 1295-1301, 2005.

[16] M.Z. Liu, S.F. Ma, and Z.W. Yang, "Stability analysis of Runge-Kutta methods for unbounded retarded differential equations with piecewise continuous arguments," Appl. Math. Comput.., vol. 191, pp. 57-66, 2007. [17] Q. Wang, Q.Y. Zhu, and M.Z. Liu, "Stability and oscillations of numerical solutions of differential equations with piecewise continuous arguments of alternately advanced and retarded type," J. Comput. Appl. Math.., vol. 235, pp. 1542-1552, 2011.

[18] J.H. He, "Variational iteration method-A kind of non-linear analytical technique: Some examples," Internat. J. Non-Linear Mech., vol. 34, no. 4, pp. 699-708, 1999.

[19] J.H. He, "Variational iteration method-Some recent results and new interpretations," J. Comput. Appl. Math.., vol. 207, no. 1, pp. 3-17, 2007.

[20] J.H. He and X.H. Wu, "Variational iteration method: New development and applications," Comput. Math. Appl., vol. 54, no.7-8, pp. 881-894, 2007.

[21] J.H. He, G.C. Wu, and F. Austin, "The variational iteration method which should be followed," Nonlinear Sci. Lett. A, vol. 1, no.1, pp. 1-30, 2010.

[22] M.M. Al-Sawalha, M.S.M. Noorani, and I. Hashim, "On accuracy of Adomian decompositionmethod for hyperchaotic Rössler system,” Chaos Solitons Fractals., vol. 40, no.4, pp. 1801-1807, 2009.

[23] M.M. Al-Sawalha and M.S.M. Noorani, "Application of the differential transformation method for the solution of the hyperchaotic Rössler system,” Comm. Non. Sci. Num. Simu., vol. 14, pp. 1509-1514, 2009.

[24] F.M. Allan, "Construction of analytic solution to chaotic dynamical systems using the Homotopy analysis method," Chaos Solitons Fractals., vol. 39, no.4, pp. 1744-1752, 2009.

[25] J.H. He, "Homotopy perturbation method for bifurcation of nonlinear problems," Int. J. Nonlinear Sci. Numer. Simu., vol. 6, pp. 207-208, 2005.

[26] J.H. He, "Some asymptotic methods for strongly nonlinear equations," Int. J. Modern Phys.B, vol. 20, no.10, pp. 1141-1199, 2006.

[27] J.H. He, "Approximate analytical solution for seepage flow with fractional derivatives in porous media," Comput. Meth. Appl. Mech. Eng., vol. 167, pp. 57-68, 1998.

[28] J.H. He, "Variational iteration method for autonomous ordinary differential systems," Appl. Math. Comput.., vol. 114, pp. 115-123, 2000.

[29] J.H. He, "An elementary introduction to recently developed asymptotic methods and nanomechanics in textile engineering," Int. J. Modern Phys.B, vol. 22, no.21, pp. 3487-3578, 2008. 